\title{
EDITORIAL
}

\section{Clinical Significance of Glycated Hemoglobin (HbA1c)}

\author{
KMHS Sirajul Haque ${ }^{1}$, *MR Siddiqui ${ }^{2}$ \\ ${ }^{1}$ Prof Dr. K.M.H.S. Sirajul Haque, Professor \& Head, Dept. of Cardiology, AKMMC \\ ${ }^{*}$ Dr. Mahmudur Rahman Siddiqui, Assistant Professor, Dept. of Medicine, AKMMC \\ * Corresponding author
}

Diabetes mellitus (DM) is now one of the most common non-communicable diseases globally. $\mathrm{DM}$ is a chronic metabolic disorder characterized by hyperglycaemia, either because the body does not produce enough insulin, or because cells do not respond to the insulin that is produced. The worldwide prevalence of diabetes in 2000 was approximately $2.8 \%$ and is estimated to grow to $4.4 \%$ by $2030 .{ }^{1}$ Magnitude of DM in Bangladesh is also increasing day by day. Prevalence of DM in our country is about $6.1 \%$ (5.6 millions) and it will hold the 8th position in the world according to the total cases of diabetes in adult population (20 to 79 years) by the year $2030 . .^{2,3}$

Diagnosis, management and regular monitoring of the DM are great challenge to both patients and physicians. Now a days a lot of treatment regimens are available. Glycated haemoglobin provides an accurate and objective measure to access the glycaemic control and also to diagnose new DM. Glycated hemoglobin is also known as glycohemoglobin, glycosylated hemoglobin or as hemoglobin A1c, HbA1c, A1C, or Hb1c (the main fraction of glycated hemoglobin). ${ }^{1}$ About 40 years ago Glycated haemoglobin (HbA1c) was initially identified as an "unusual" haemoglobin in diabetic patients. ${ }^{4}$ HbAlc was first separated from other forms of hemoglobin by Huisman and Meyering in the year 1958.5 It was characterized as a glycoprotein by Bookchin and Gallop in 1968. ${ }^{6}$ Samuel Rahbar et al. in 1969 first described its relationship with diabetes. ${ }^{7}$ Glycated hemoglobins are characteristically Ketoamine, formed through a two-step nonenzymatic pathway between hemoglobin and blood glucose. Blood glucose and hemoglobin are combined to form aldimine as a first step, which is a reversible form. In the second step, this labile aldimine is slowly converted to Ketoamine form which is stable and irreversible. ${ }^{1,8,9} \mathrm{In}$

AKMMC J 2013; 4(1): 3-5 individuals with poorly controlled diabetes, the quantities of these glycated hemoglobins are much higher than in healthy people. ${ }^{9}$ The HbA1c level is directly proportional to average blood glucose concentration over the previous 4 weeks to 3 months or the average lifespan of the erythrocyte. However, it is important to remember that $\mathrm{HbA} 1 \mathrm{c}$ only reflects glucose concentrations over 4 to 8 weeks provided there is a normal hemoglobin concentration and normal red blood cell survival. ${ }^{1,10}$

Total glycated hemoglobin (Total GHb) refers to all the glycated hemoglobins, including glycated hemoglobin variants. Total glycated hemoglobin is usually determined by affinity chromatography or immunassays. HbA1c is the major subfraction of the glycated normal hemoglobin (HbA1). HbA1c is usually determined by ion-exchange high-performance liquid chromatography (HPLC) or gel electrophoresis. ${ }^{9}$ Excessive formation of early glycation products may adversely affect several functions of blood vessels, lipid metabolism and prone to develop diabetic complications. In blood vessels, uptake of LDL may be enhanced; resulting in atherogenesis and also increases the free radical mediated damage. These reversible biochemical abnormalities probably play a role in the pathogenesis of the early functional changes in the diabetic microvasculature. ${ }^{1,11}$ Higher amounts of HbA1c in diabetic patients, indicating poorer control of blood glucose levels, have been associated with diabetic complications like; cardiovascular disease, nephropathy, and retinopathy. ${ }^{1,12}$

HbA1c was introduced into clinical use in the 1980s and subsequently has become an important part of clinical practice. ${ }^{12}$ The real breakthroughs for the clinical use of HbA1c in 
diabetes came from the large prospective DCCT (Diabetes Control and Complications Trial) and UKPDS (United Kingdom Prospective Diabetes Study) studies showing that glycaemic control, by the measured of HbA1c levels, was related to risk of developing the microvascular complications of diabetes. HbA1c reflects average plasma glucose over the previous eight to 12 weeks. ${ }^{1,10,12}$ The rate of formation of $\mathrm{HbA} 1 \mathrm{c}$ is directly proportional to the ambient blood glucose concentration; a rise of $1 \%$ in $\mathrm{HbA} 1 \mathrm{c}$ corresponds to an approximate average increase of $2 \mathrm{mmol} / \mathrm{L}$ ( $36 \mathrm{mg} / \mathrm{dL})$ in blood glucose.

HbA1c has now been recommended by an International Committee and by the ADA (American Diabetes Association) as a means to diagnose diabetes and as a screening test for persons at high risk of diabetes. ${ }^{12,13}$ The 2010 American Diabetes Association Standards of Medical Care in Diabetes added the A1c $\geq 48$ $\mathrm{mmol} / \mathrm{mol}(\geq 6.5 \%)$ as another criterion for the diagnosis of diabetes. ${ }^{14}$ The International Diabetes Federation and American College of Endocrinology recommend $\mathrm{HbA1c}$ values below $48 \mathrm{mmol} / \mathrm{mol}(6.5 \%) .{ }^{13} \mathrm{An} \mathrm{HbA1c}$ of $6.5 \%$ is recommended as the cut point for diagnosing diabetes but a value of less than $6.5 \%$ does not exclude diabetes diagnosed using glucose tolerance tests. ${ }^{13}$ Diagnosis of gestational diabetes still requires fasting and glucose tolerance measurements and not the glycated hemoglobin. ${ }^{13,14} \mathrm{HbA1c}$ levels decrease during the second trimester of a normal nondiabetic pregnancy and rise during the third trimester. ${ }^{15}$

It can be performed at any time of the day and does not require any special preparation such as fasting. These properties have made it the preferred test for assessing glycaemic control in people with diabetes. ${ }^{13}$ The American Diabetes Association guidelines suggest that the HbA1c test can be performed at least two times a year in patients with diabetes that are meeting treatment goals (and that have stable glycemic control) and quarterly in patients with diabetes whose therapy has changed or that are not meeting glycemic goals. ${ }^{16}$ Results of $\mathrm{HbA1c}$ can be unreliable in many circumstances, such as after blood loss, for example, after surgery, blood transfusions, anemia, or high erythrocyte turnover; in the presence of chronic renal or liver disease; after administration of high-dose vitamin $\mathrm{C}$; or erythropoietin in treatment. ${ }^{10}$ Lower-thanexpected levels of HbA1c can be seen in people with shortened red blood cell lifespan, such as with glucose-6-phosphate dehydrogenase deficiency, sickle-cell disease, or any other condition causing premature red blood cell death. On the converse, higher-than-expected levels can be seen in people with a longer red blood cell lifespan, such as with Vitamin B12 or folate deficiency. ${ }^{17}$

Table I: Some of the factors that influence HbA1c and its measurement.(Gallagher et al) 12,18

\section{Erythropoiesis}

Increased HbAlc: iron, vitamin B12 deficiency, decreased erythropoiesis.

Decreased HbAlc: administration of erythropoietin, iron, vitamin $\mathrm{B} 12$, reticulocytosis, chronic liver disease.

\section{Altered Haemoglobin}

Genetic or chemical alterations in haemoglobin: haemoglobinopathies, $\mathrm{HbF}$, methaemoglobin, may increase or decrease HbA1c.

\section{Glycation}

Increased HbA1c: alcoholism, chronic renal failure, decreased intraerythrocyte $\mathrm{pH}$.

Decreased HbAlc: aspirin, vitamin $\mathrm{C}$ and $\mathrm{E}$, certain haemoglobinopathies, increased intra-erythrocyte $\mathrm{pH}$.

Variable HbA1c: genetic determinants.

\section{Erythrocyte destruction}

Increased HbAlc: increased erythrocyte life span: Splenectomy.

Decreased A1c: decreased erythrocyte life span: haemoglobinopathies, splenomegaly, rheumatoid arthritis or drugs such as antiretrovirals, ribavirin and dapsone.

\section{Assays}

Increased HbAlc: hyperbilirubinaemia, carbamylated haemoglobin, alcoholism, large doses of aspirin, chronic opiate use.

Variable HbAlc: haemoglobinopathies.

Decreased HbAlc: hypertriglyceridaemia.

Table II: Diagnostic criteria for diabetes (WHO report 2011) ${ }^{12}$

HbA1c can be used as a diagnostic test for diabetes provided that stringent quality assurance tests are in place and assays are standardized to criteria aligned to the international reference values, and there are no conditions present which preclude its accurate measurements

An HbA1c of $6.5 \%$ was recommended as the cut-off point for diagnosing diabetes. A value $<6.5 \%$ does not exclude diabetes diagnosed using glucose tests

Summary of tests that can be used

Fasting plasma glucose : ${ }^{\geq} 7.0 \mathrm{mmol} \mathrm{L}-1$ (126 mg dL-1) or

2-h plasma glucosea : $\geq_{11.1 \mathrm{~mol} \mathrm{~L}-1 \text { (200 mg dL-1) or }}$ HbAlc : ${ }^{6.5 \%}$

a $75 \mathrm{~g}$ oral glucose tolerance test 


\section{Conclusion}

Over the last four decades HbA1c comes out with many facts of diabetes and now it is a very important part of both diagnosis and treatment of diabetes. However, HbA1c may be affected by a variety of genetic, physiological, haematological and illness-related factors but another major factor concerns costs and availability of HbA1c assays in many countries.

\section{References}

1. Sultanpur CM, Deepa K, Kumar SV. Comprehensive review on hba1c in diagnosis of diabetes mellitus. International Journal of Pharmaceutical Sciences Review and Research 2010; 3(2): 119-22.

2. Diabetes Atlas 2009, IDF, Belgium.

3. Text Book of Diabetes, 3rd edition- vol 1, edited by John C. Pickup and G. Williams, Blackwell Science, 2003, p 5.1-6.14.

4. Rahbar, Blumenfeld O, Ranney HM. Studies of an unusual haemoglobin in patients with diabetes mellitus. Biochem Biophys Res Commun 1969; 36: 838-43.

5. Huisman TH, Martis EA, Dozy A. "Chromatography of hemoglobin types on carboxymethylcellulose". J. Lab. Clin. Med. 1958; 52 (2): 312-27. PMID 13564011.

6. Bookchin RM, Gallop PM. "Structure of hemoglobin A1c: nature of the N-terminal beta chain blocking group". Biochem. Biophys. Res. Commun. 1968;32 (1):86-93. DOI:10.1016/0006-291X(68)90430-0. PMID 4874776 .

7. Rahbar S, Blumenfeld O, Ranney HM. "Studies of an unusual hemoglobin in patients with diabetes mellitus". Biochem. Biophys. Res. Commun. 1969; 36 (5): 838-43. DOI:10.1016/0006-291X(69)90685-8. PMID 5808299.

8. John Pick Up and Gareth Williams, text book of Diabetes, published by Blackwell science 1998; 2: 5.6-5.7.
9. Weykamp C, John WG, Mosca A. A Review of the Challenge in Measuring Hemoglobin A1c. J Diabetes Sci Technol 2009; 3(3): 439-45.

10. Nathan DM, Turgeon H, Regan S. Relationship between glycated haemoglobin levels and mean glucose levels over time. Diabetologia 2007; 50: 2239-44.

11. Gillery P, Monboisse JC, Maquart FX, et al. Glyation of proteins as a source of superoxide. Diab metab 1988; 14: 25-30.

12. Use of Glycated Haemoglobin (HbA1c) in the Diagnosis of Diabetes Mellitus: Abbreviated Report of a WHO Consultation. World Health Organization 2011.

13. International Expert Committee report on the role of the A1c assay in the diagnosis of diabetes. Diabetes Care 2009; 32(7): 1327-34. DOI:10.2337/dc09-9033. PMC 2699715.PMID 19502545.

14. "Executive summary: Standards of medical care in diabetes-2010". Diabetes Care 2010; 33 (Suppl 1): S410. DOI: $10.2337 / \mathrm{dc} 10-S 004$. PMC 2797389.PMID 20042774.

15. Nitin S. HbA1c and factors other than diabetes mellitus affecting it. Singapore Med J 2010; 51(8): 616-22.

16. American Diabetes Association (2007). "Standards of medical care in diabetes--2007". Diabetes Care 2007; 30 (Suppl 1): S4-S41. DOI: 10.2337/dc07-S004. PMID 17192377.

17. Kilpatrick ES, Bloomgarden ZT, Zimmet PZ. "Is haemoglobin A1c a step forward for diagnosing diabetes?" BMJ 2009; 339: b4432. DOI: 10.1136/bmj.b4432. PMID 19903702.

18. Gallagher EJ, Bloomgarder ZT, Le Roith D. Review of hemoglobin A1c in the management of diabetes. Journal of Diabetes 2009; 1: 9-17. 